\title{
OPTIMAL OBSERVABILITY FOR CONTINUOUS PETRI NETS
}

\author{
Cristian Mahulea, Laura Recalde, Manuel Silva
}

\author{
Dep. de Informática e Ingeniería de Sistemas, Centro \\ Politécnico Superior, Universidad de Zaragoza, María de \\ Luna 3, E-50015 Zaragoza, Spain, \\ $\{$ cmahulea,lrecalde,silva\}@unizar.es
}

\begin{abstract}
Optimal observability of continuous Petri Nets consists in deciding the places to be measured (considering that all are measurable) such that the net system is observable and a cost function is minimal. Unfortunately this is not a simple covering problem. The results obtained in the paper are used in the implementation of an algorithm to improve the pure combinatorial search. Copyright ${ }^{\circledR} 2005$ IFAC
\end{abstract}

Keywords: Petri-nets, Continuous systems, Optimal Estimation, Algorithms.

\section{INTRODUCTION}

Petri nets represent a powerful formalism for modelling discrete event concurrent systems. Stochastic T-timed Petri nets under infinite servers semantics are well-known performance evaluation models (Ajmone Marsan et al., 1995). Under high traffic or heavy loads discrete event systems often suffer from state explosion. One way to tackle this problem is to relax the original discrete model.

Fluidification is a relaxation technique in which discrete elements of the system are "taken" as continuous. As in other discrete event formalisms (e.g. queueing networks), the continuous relaxation of Petri nets has been introduced in order to deal with the state explosion problem. Under infinite servers semantics, a timed continuous Petri net system, obtained as a first-order relaxation, can be seen as a deterministic piecewise linear system (Silva and Recalde, 2002; Silva and Recalde, 2004). That is the evolution of the state of the system is ruled by a set of switching noisefree linear differential equation systems. Hence, there exists the chance of applying some results coming from Systems Theory to continuous Petri

This work was partially supported by project CICYT and FEDER TIC2001-1819 and DPI2003-06376. nets. Timed continuous Petri net systems have the particularity that, at any given instant, the timeinvariant linear system differential equations that rule its evolution depend uniquely on the state of the system (marking). Hence, the switch from one linear differential equation system to another one is activated by an internal event, i.e., by a certain change in the marking of the system.

The differential equations describing a continuous dynamic system can be presented as a state space model; the states are defined by means of state variables. Some of them can be directly measured, while, under some conditions, others can be estimated. The estimation constitutes the observation. In this paper we consider that all marking variables can be measured but at different cost, the cost of its sensor.

The main goal of this paper is to develop an algorithm for optimal choice (minimum cost) of the places that must be measured (sensed) in order to ensure the observability of the system.

The paper is structured as follows: In Section 2 continuous Petri nets are recalled, while Section 3 presents the observability problem for continuous Petri nets. Optimal observability problem is stated in Section 4. Section 5 shows the main results obtained and a computational algorithm is illustrated at the end. 


\section{CONTINUOUS PETRI NET SYSTEMS}

\subsection{Untimed Continuous Petri Net Systems}

The reader is assumed to be familiar with Petri nets $(P N s)$ (see for example (Murata, 1989)). The $P N s$ that will be considered are continuous, relaxation of discrete ones. Unlike 'usual' discrete systems, the amount in which a transition can be fired in a continuous Petri net system is not restricted to be a natural number. The marking of the system becomes a vector of real numbers. A $P N$ system is a pair $\left\langle\mathcal{N}, \mathbf{m}_{\mathbf{0}}\right\rangle$, where $\mathcal{N}$ specifies the net structure, $\mathcal{N}=\langle P, T$, Pre, Post $\rangle$ and $\mathbf{m}_{\mathbf{0}}$ is the initial marking. The sets of places and transitions are denoted by $P$ and $T$ respectively. Matrices Post and Pre are the arc weight matrices and $\mathbf{C}=$ Post - Pre is the token flow matrix. The set of input (output) places of a given set of transitions $V$ is denoted as ${ }^{\bullet} V\left(V^{\bullet}\right)$. Analogously, the set of input (output) transitions of a given set of places $W$ is denoted as ${ }^{\bullet} W\left(W^{\bullet}\right)$.

In continuous Petri net systems a transition $t$ is enabled at a marking $\mathbf{m}$ iff every input place of $t$ is marked (for every $p \in \bullet^{\bullet} t, \mathbf{m}[p]>0$ ). As in discrete systems, the enabling degree at marking $\mathbf{m}$ of a transition measures the maximum amount in which the transition can be fired in a single occurrence, i.e., $\operatorname{enab}(t, \mathbf{m})=\min _{p \in \bullet}\{\mathbf{m}[p] / \operatorname{Pre}[p, t]\}$. The firing of $t$ in an amount $\alpha \leq \operatorname{enab}(t, \mathbf{m})$ produces a new marking $\mathbf{m}^{\prime}$, and it is denoted as $\mathbf{m} \stackrel{\alpha t}{\longrightarrow} \mathbf{m}^{\prime}$. It holds $\mathbf{m}^{\prime}=\mathbf{m}+\alpha \cdot \mathbf{C}[P, t]$, hence, as in discrete systems the state equation $\mathbf{m}=\mathbf{m}_{\mathbf{0}}+$ $\mathbf{C} \cdot \boldsymbol{\sigma}$ summarizes the way the marking evolves, where $\boldsymbol{\sigma} \in\left(R^{+}\right)^{|P|}$ is the firing count vector. As in discrete nets, continuous nets can be classified according to their structure:

- $\mathcal{N}$ is a weighted T-graph if $\forall p \in P:\left|p^{\bullet}\right|=$ $|\bullet p|=1$.

- $\mathcal{N}$ is Choice-Free (CF) if $\forall p \in P:\left|p^{\bullet}\right| \leq 1$.

- $\mathcal{N}$ is Join-Free (JF) if $\forall t \in T:|\bullet t| \leq 1$.

- $\mathcal{N}$ is Equal Conflict (EQ) if $\bullet t \cap \bullet t^{\prime} \neq \emptyset \Rightarrow$ $\operatorname{Pre}[P, t]=\operatorname{Pre}\left[P, t^{\prime}\right]$.

- $\mathcal{N}$ is Attribution Free (AF) if $\forall p \in P:\left|{ }^{\bullet} p\right| \leq 1$.

\subsection{Timed Continuous Petri Net Systems}

For the timing interpretation, a first order (or deterministic) approximation of the discrete case (Recalde and Silva, 2001) will be used, assuming that the delays associated to the firing of the transitions can be approximated by their mean values. Each transition $t$ has associated an internal firing speed $\boldsymbol{\lambda}>0$. The state equation has an explicit dependence on time $\mathbf{m}(\tau)=\mathbf{m}_{\mathbf{0}}+\mathbf{C} \cdot \boldsymbol{\sigma}(\tau)$. Deriving with respect to time, $\dot{\mathbf{m}}(\tau)=\mathbf{C} \cdot \dot{\boldsymbol{\sigma}}(\tau)$ is obtained. Let us denote $\mathbf{f}=\dot{\boldsymbol{\sigma}}$, since it represents the flow through the transitions. In this paper it will be assumed that every transition has at least one input place. Infinite servers semantics will be considered. Under this semantics, the flow of a transition is given by the product of $\boldsymbol{\lambda}$ and its enabling degree, i.e., $\mathbf{f}[t]=\boldsymbol{\lambda} \cdot \operatorname{enab}(t, \mathbf{m})=$ $\boldsymbol{\lambda} \cdot \min _{p \in{ }^{*} t}\{\mathbf{m}[p] /$ Pre $[p, t]\}$, what leads to a nonlinear system.
In JF nets, the computation of the enabling degrees does not require the min operator. Hence, the flow of the transitions can be expressed as $\mathbf{f}=\boldsymbol{\Psi} \cdot \mathbf{m}$ where $\boldsymbol{\Psi}[t, p]=\boldsymbol{\lambda}_{t} / \operatorname{Pre}[p, t]$ if $p=\bullet t$, $\boldsymbol{\Psi}[t, p]=0$ otherwise. Consequently, the evolution of the marking can be described by an equation in the form $\dot{\mathbf{m}}=\mathbf{C} \cdot \mathbf{f}=\mathbf{A} \cdot \mathbf{m}$, where $\mathbf{A}=\mathbf{C} \cdot \mathbf{\Psi}$. Hence, a JF system can be interpreted as a (nonnegative) linear system.

For a general $P N$ system, matrix A (usually, not of maximal rank) is piecewise-constant, depending on $\mathbf{m}$. To compute $\mathbf{A}(\mathbf{m})$, it is necessary to know the set of places that are actually constraining the enabling of the transitions, i.e., the set of places that is giving the minimum in the expression for the enabling degree. Once this set is computed, it is easy to establish a linear relationship between the marking of the places in this set and the flow of the transitions: $\dot{\mathbf{m}}=\mathbf{A}(\mathbf{m}) \cdot \mathbf{m}$, with $\mathbf{A}(\mathbf{m})=\mathbf{C} \cdot \boldsymbol{\Psi}$ where $\boldsymbol{\Psi}[t, p]=\boldsymbol{\lambda}_{t} / \operatorname{Pre}[p, t]$ if $p \in$ $\bullet t$ and $\mathbf{m}[p] / \operatorname{Pre}[p, t]=\min _{q \in \bullet}\{\mathbf{m}[q] / \operatorname{Pre}[q, t]\}$, $\boldsymbol{\Psi}[t, p]=0$ otherwise.

Definition 1. (Júlvez et al., 2004) Given a $P N$ system, the $P T$-set at marking $\mathbf{m}$ is defined as: $\mathrm{PT}$-set $(\mathbf{m})=\left\{(p, t) \mid \mathbf{f}[t]=\boldsymbol{\lambda}_{t} \cdot \mathbf{m}[p] / \operatorname{Pre}[p, t]\right\}$.

A continuous Petri net system can be seen as a time-invariant piecewise linear system in which the switches among the linear systems are activated by internal events, i.e., the change from one PT-set to another does not need any external agent, just a certain change in the system marking. The marking of the system and its first derivative with respect to time are continuous.

\section{OBSERVABILITY: PROBLEM STATEMENT}

Let us briefly consider first linear time invariant systems, for which observability has been thoroughly studied (see, for example, (Luenberger, 1971; Ogata, 1995)). An unforced linear system (i.e., without inputs) is usually expressed by equations $\dot{\mathbf{x}}(t)=\mathbf{A} \cdot \mathbf{x}(t), \mathbf{y}(t)=\mathbf{S} \cdot \mathbf{x}(t)$ where $\mathbf{x}(\mathrm{t})$ is the state of the system and $\mathbf{y}(\mathrm{t})$ is the output, i.e., the set of measured variables. Knowing matrices $\mathbf{A}$ and $\mathbf{S}$ and being able to watch the evolution of $\mathbf{y}(\mathrm{t})$, a linear system is said to be observable iff it is possible to compute its initial state, $\mathbf{x}\left(t_{0}\right)$ (in fact, since the system is deterministic, knowing the state at the initial time is equivalent to knowing the state at any time).

In Systems Theory a very well-known observability criterion exists which allows to decide whether a continuous linear system is observable or not. Besides, several approaches exist to compute the initial state of a continuous time linear system that is observable.

Given an unforced linear system, $\dot{\mathbf{x}}(t)=\mathbf{A}$. $\mathbf{x}(t), \mathbf{y}(t)=\mathbf{S} \cdot \mathbf{x}(t)$ the output of the system $(\mathbf{y}(t))$ and the observability matrix $(\vartheta)$ are:

$$
\begin{gathered}
\mathbf{y}(t)=\mathbf{S} \cdot e^{A \cdot t} \cdot \mathbf{x}\left(t_{0}\right) \\
\vartheta=\left(\mathbf{S}^{T},(\mathbf{S A})^{T}, \cdots,\left(\mathbf{S A}^{n-1}\right)^{T}\right)^{T}
\end{gathered}
$$




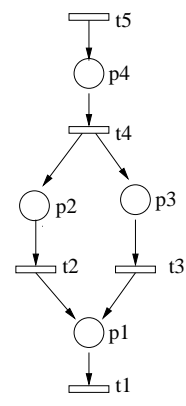

(a)

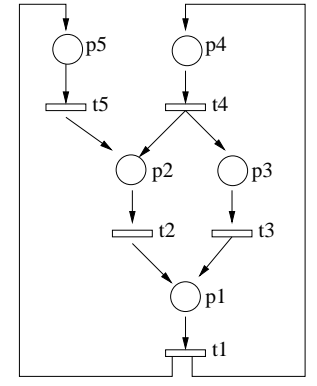

(b)
Fig. 1. Two JF nets: (a) whose marking cannot be estimated from the observation of $p_{1}$ if $\boldsymbol{\lambda}_{2}=\boldsymbol{\lambda}_{3}$; (b) not observable measuring $p_{5}$ if $\boldsymbol{\lambda}_{4}=\boldsymbol{\lambda}_{5}=\frac{2 \cdot \boldsymbol{\lambda}_{2} \cdot \boldsymbol{\lambda}_{3}}{\boldsymbol{\lambda}_{2}+\boldsymbol{\lambda}_{3}}$.

Proposition 2. (Luenberger, 1971) Equation (1) is soluble $\forall \mathbf{x}\left(t_{0}\right), \forall t \geq 0$ iff observability matrix $\vartheta$ has full rank.

An interpretation of complete observability is that there is no simplification in the transfer function between the state variables and the output (Sinha, 1984). Considering a single-input / single-output system, the transfer function between the state variables and the output is given by:

$$
\mathcal{Y}(s)=\mathbf{S}(s I-A)^{-1}=\frac{1}{\Delta(s)}\left[q_{1}(s) \ldots q_{n}(s)\right]
$$

If $\mathcal{Y}(\mathrm{s})$ has a cancellation (all the polynomials $q_{i}(s)$ and $\left.\Delta(s)\right)$ have a common factor) this cancelled state cannot be observed in the output $\mathbf{y}$.

Definition 3. (Júlvez et al., 2004) Let $\left\langle\mathcal{N}, \boldsymbol{\lambda}, \mathbf{m}_{\mathbf{0}}\right\rangle$ be a continuous Petri net system, $\mathcal{D}$ the set of measured places.

- A place $p \in P$ is observable from $\mathcal{D}$ iff $\mathbf{m}_{\mathbf{0}}[p]=\mathbf{m}\left(\tau_{0}\right)[p]$ can be computed by measuring the marking evolution of the places in $\mathcal{D}$. Let $\mathbf{O}$ be the set of all places observable from $\mathcal{D}$.

- $\left\langle\mathcal{N}, \boldsymbol{\lambda}, \mathbf{m}_{\mathbf{0}}\right\rangle$ is observable from $\mathcal{D}$ iff every place $p \in P$ is observable $(\mathbf{O}=P)$.

Straightforward applycation of Prop. 2, leads to:

Property 4. Given a Petri net system and $\Gamma_{i}$ the linear system associated to PT-set $i$. The PT-set $i$ is observable iff its associated observability matrix $\vartheta_{i}$ has full rank.

\section{OPTIMAL OBSERVABILITY PROBLEM}

Let us now assume that places have associated a measuring cost, $\forall p \in P: w(p)>0$. The observability cost for a given set $\mathcal{D}$ is $w(\mathcal{D})=$ $\sum_{p_{i} \in \mathcal{D}} w\left(p_{i}\right)$; the problem is to determine a set $\mathcal{D}$ with minimum cost from which $\left\langle\mathcal{N}, \boldsymbol{\lambda}, \mathbf{m}_{\mathbf{0}}\right\rangle$ is observable.

In general, if for a set of measured places $\mathcal{D}_{i}$ the observable places are $\mathbf{O}_{i}$ then for the set $\mathcal{D}=\mathcal{D}_{1} \cup \mathcal{D}_{2} \cup \cdots \cup \mathcal{D}_{i}$ the observable places are $\mathbf{O}$, with $|\mathbf{O}| \geq\left|\mathbf{O}_{1} \cup \mathbf{O}_{2} \cup \cdots \cup \mathbf{O}_{i}\right|$. Considering Fig. 1(a) with $\boldsymbol{\lambda}_{2}=\boldsymbol{\lambda}_{3}$, the set of all places observable from $\mathcal{D}_{1}=\left\{p_{1}\right\}$ is $\mathbf{O}_{1}=\left\{p_{1}\right\}$ and from $\mathcal{D}_{2}=\left\{p_{2}\right\}$ is $\mathbf{O}_{2}=\left\{p_{2}, p_{4}\right\}$ but, measuring $\mathcal{D}=\mathcal{D}_{1} \cup \mathcal{D}_{2}=\left\{p_{1}, p_{2}\right\}$, the system is observable, so $\mathbf{O}=\left\{p_{1}, p_{2}, p_{3}, p_{4}\right\}$ and $|\mathbf{O}|>\left|\mathbf{O}_{1} \cup \mathbf{O}_{2}\right|$.

Assuming that sets $\mathcal{D}_{i}$ and $\mathbf{O}_{i}$ are determined, optimal observability problem can be seen as a Set Covering Problem (SCP), which is NP-hard in the strong sense (Garey and Johnson, 1979). Unfortunately, the number of covering elements do not restrict to the number of places, $\mathcal{D}_{i}=\left\{p_{i}\right\}$, what makes practical complexity very important and forces us to look for some properties of $P N s$.

\section{OPTIMAL OBSERVABILITY FOR PNS}

\subsection{General nets: reduction to JF and $C F(J C-F)$}

Consider first Fig.2(a). In the absence of any information about the markings of $p_{1}$ and $p_{2}$, it is observable iff all places are measured.

The place $p_{3}$ must be measured: using the marking of $p_{3}$, the flow of the transition $t_{3}$ can be computed as $f_{3}=\boldsymbol{\lambda}_{3} \cdot \mathbf{m}\left[p_{3}\right]$. The derivative of the marking and the flow of transition $t_{3}$ permit the computation of the flow of transition $t$ using $f_{t}=\dot{\mathbf{m}}\left[p_{3}\right]+f_{3}$. This flow is equal by the other hand with $f_{t}=\boldsymbol{\lambda}_{t} \cdot \min \left(\mathbf{m}\left[p_{1}\right], \mathbf{m}\left[p_{2}\right]\right)$. In the last expression, $f_{t}$ and $\boldsymbol{\lambda}_{t}$ are known which implies that the minimum between $\mathbf{m}\left[p_{1}\right]$ and $\mathbf{m}\left[p_{2}\right]$ can be evaluated. If always $\mathbf{m}\left[p_{1}\right] \leq \mathbf{m}\left[p_{2}\right]$ (so the place $p_{2}$ is time implicit), $\mathbf{m}\left[p_{1}\right]$ equals with the minimum and $p_{2}$ must be measured. Identically, if $\mathbf{m}\left[p_{2}\right] \leq \mathbf{m}\left[p_{1}\right]$ in any moment, $p_{1}$ should be measured. If no information regarding $\mathbf{m}\left[p_{1}\right]$ and $\mathbf{m}\left[p_{2}\right]$ is given then the only solution for the observability is to measure both $p_{1}$ and $p_{2}$.

Therefore, the flow of transition $t$ gives the minimum marking of the input places. Considering any initial marking, this minimum does not provide enough information to estimate $\mathbf{m}\left[p_{1}\right]$ or $\mathbf{m}\left[p_{2}\right]$, so the arc between $p_{1}$ and $t$ and the arc between $p_{2}$ and $t$ can be deleted without affecting the observability. The same considerations can be used to prove the next theorem.

Theorem 5. Let $\mathcal{N}$ be a Petri net, and let $\mathcal{N}^{\prime}$ be the net obtained removing all the input arcs of the synchronizations. $\left\langle\mathcal{N}, \boldsymbol{\lambda}, \mathbf{m}_{0}\right\rangle$ is observable for any initial marking iff $\left\langle\mathcal{N}^{\prime}, \boldsymbol{\lambda}^{\prime}, \mathbf{m}_{0}^{\prime}\right\rangle$ is observable.

The net $\mathcal{N}^{\prime}$ obtained by eliminating all the synchronizations is a JF. All the conflicts are EQ and so, the net can be mapped into CF. Thus any criteria developed for JC-F PNs is immediately extended to all $P N s$.

\subsection{Attribution Free Petri nets}

For JF \& CF \& AF (JCA-F) $P N$, the optimal observability is resolved without difficulty.

Proposition 6. (Júlvez et al., 2004) Let $\mathcal{N}$ be a continuous JCA-F Petri net and $p$ a measured place. Let $p^{\prime}$ be a place such that a path from $p^{\prime}$ to $p$ exists, then $p^{\prime}$ is structurally observable (the 
marking of $p^{\prime}$ can be computed using the marking of $p$ for any $\boldsymbol{\lambda}$, the firing rates of the transitions).

The marking of place $p^{\prime}$ can be computed going backwards on the path. Beginning with place $p$, in each step the marking of a previous place is computed.

Corollary 7. Let $\left\langle\mathcal{N}, \boldsymbol{\lambda}, \mathbf{m}_{0}\right\rangle$ be a weighted $\mathrm{T}$ system. Then $\left\langle\mathcal{N}, \boldsymbol{\lambda}, \mathbf{m}_{0}\right\rangle$ is observable for any initial marking iff all synchronization places are measured or, in the case of weighted cycle, one arbitrary place.

Definition 8. Let $\mathcal{N}$ be a continuous Petri net. The set of places $\mathcal{F}=\left\{p \in \mathcal{N} \mid p^{\bullet}=\emptyset\right.$ or $\left(p^{\bullet}\right)^{\bullet}=$ $\emptyset\}$ is called set of final places.

Theorem 9. Let $\left\langle\mathcal{N}, \boldsymbol{\lambda}, \mathbf{m}_{\mathbf{0}}\right\rangle$ be a JCA-F Petri net system. If $\mathcal{F} \neq \emptyset$, minimum cost of the observability is $w(\mathcal{F})$; otherwise the system is a cycle and measuring the place with minimum cost guarantees observability.

Proof: Assume first that $\mathcal{N}$ is not a cycle. The final places are essential covers so must be included in the solution; obviously there is a backward path from a final place to any place of the $P N$ and, according with Prop. (6), the marking of all places can be evaluated.

When $\mathcal{F}$ is empty, the system is a cycle (Teruel et $a l ., 1997)$ and measuring one place the system is observable.

\subsection{General nets (JC-F case):nets with attribution}

The observability problem of general nets was reduced to that of JC-F nets in Sect. 5.1. The remaining problem is to deal with attributions because they introduce zeros in the transfer function that can produce a cancellation.

Let us now consider the JC-F Petri net in Fig.1(a) (it has an attribution in $p_{1}$ ). Assume that $p_{1}$ is measured. This system is a continuous timeinvariant linear system. If we consider that the input of the system is $f_{5}$ and the output $f_{1}$, the equivalent linear system $\dot{\mathbf{x}}(t)=\mathbf{A} \cdot \mathbf{x}(t)+\mathbf{B}$. $\mathbf{u}(t), \mathbf{y}(t)=\mathbf{S} \cdot \mathbf{x}(t)$ has:

$$
\begin{gathered}
\mathbf{A}=\left(\begin{array}{cccc}
-\boldsymbol{\lambda}_{1} & \boldsymbol{\lambda}_{2} & \boldsymbol{\lambda}_{3} & 0 \\
0 & -\boldsymbol{\lambda}_{2} & 0 & \boldsymbol{\lambda}_{4} \\
0 & 0 & -\boldsymbol{\lambda}_{3} & \boldsymbol{\lambda}_{4} \\
0 & 0 & 0 & -\boldsymbol{\lambda}_{4}
\end{array}\right), \\
\mathbf{B}=\left(\begin{array}{llll}
0 & 0 & 0 & 1
\end{array}\right)^{T}, \mathbf{S}=\left(\begin{array}{llll}
\boldsymbol{\lambda}_{1} & 0 & 0 & 0
\end{array}\right)
\end{gathered}
$$

The transfer function vector between the state variables and the output, using Equation (3) is:

$$
\begin{gathered}
\mathcal{Y}(s)=\frac{\boldsymbol{\lambda}_{1}}{\left(s+\boldsymbol{\lambda}_{1}\right)\left(s+\boldsymbol{\lambda}_{2}\right)\left(s+\boldsymbol{\lambda}_{3}\right)\left(s+\boldsymbol{\lambda}_{4}\right)} H^{T} \\
H=\left[\begin{array}{c}
\left(s+\boldsymbol{\lambda}_{2}\right) \cdot\left(s+\boldsymbol{\lambda}_{3}\right) \cdot\left(s+\boldsymbol{\lambda}_{4}\right) \\
\boldsymbol{\lambda}_{2} \cdot\left(s+\boldsymbol{\lambda}_{3}\right) \cdot\left(s+\boldsymbol{\lambda}_{4}\right) \\
\boldsymbol{\lambda}_{3} \cdot\left(s+\boldsymbol{\lambda}_{2}\right) \cdot\left(s+\boldsymbol{\lambda}_{4}\right) \\
\boldsymbol{\lambda}_{4} \cdot\left(\boldsymbol{\lambda}_{2} \cdot\left(s+\boldsymbol{\lambda}_{3}\right)+\boldsymbol{\lambda}_{3} \cdot\left(s+\boldsymbol{\lambda}_{2}\right)\right)
\end{array}\right]
\end{gathered}
$$

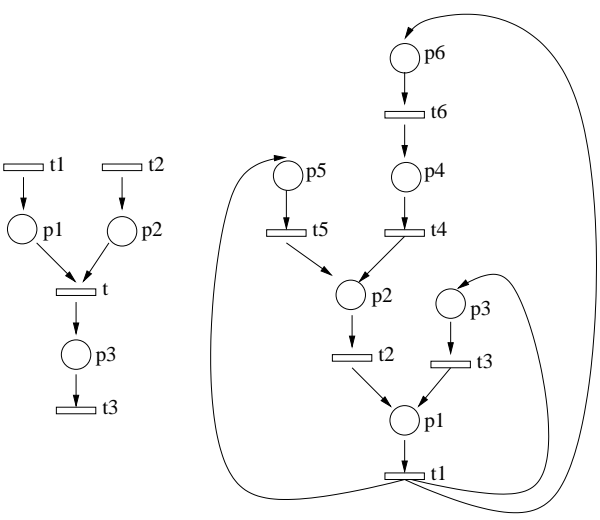

(a)

Fig. 2. (a) A $P N$ observable for any initial marking if all places are measured; (b) JF net, not observable measuring $p_{5}$ or $p_{3}$ but observable measuring both $\left(\boldsymbol{\lambda}_{3}=\boldsymbol{\lambda}_{4}\right.$ and $\left.\boldsymbol{\lambda}_{5}=\boldsymbol{\lambda}_{6}\right)$.

In Equation (5), if $\boldsymbol{\lambda}_{2}=\boldsymbol{\lambda}_{3}$ there is a pole-zero simplification in all elements of vector $\mathcal{Y}(s)$ that leads to the conclusion that the system is not observable (Sinha, 1984). If $\boldsymbol{\lambda}_{4}=\frac{2 \cdot \boldsymbol{\lambda}_{2} \cdot \boldsymbol{\lambda}_{3}}{\boldsymbol{\lambda}_{2}+\boldsymbol{\lambda}_{3}}$ there is another simplification and the system is not observable. Consequently, observability is a global property for an attribution.

Returning to the $P N$ in Fig.1(a), $p_{1}$ should be measured. If $\boldsymbol{\lambda}_{2}=\boldsymbol{\lambda}_{3}$ we are forced to measure $p_{2}$ or $p_{3}$ and obviously we should choose the one with minimum cost. Unfortunately this simple rule cannot be always applied. For example, the net presented in Fig.1(b) with $\boldsymbol{\lambda}_{2} \neq \boldsymbol{\lambda}_{3}$ and $\boldsymbol{\lambda}_{4}=\boldsymbol{\lambda}_{5}=\frac{2 \cdot \boldsymbol{\lambda}_{2} \cdot \boldsymbol{\lambda}_{3}}{\boldsymbol{\lambda}_{2} \cdot \boldsymbol{\lambda}_{3}}$ is observable if $p_{4}$ is measured, but is not observable if the measured place is $p_{5}$ although these places make an attribution to $p_{2}$.

Clearly, if after eliminating all the synchronizations and measuring all places in $\mathcal{F}$, the observability matrix has full rank, $\mathcal{F}$ is the solution. If not, it means that there are attributions that are not observable and we should measure more places to obtain an observable system. Unfortunately, the selection of extra places is difficult and in practice may be an exponential problem.

Let us consider the net in Fig.2(b) and assume $\boldsymbol{\lambda}_{3}=\boldsymbol{\lambda}_{4}, \boldsymbol{\lambda}_{5}=\boldsymbol{\lambda}_{6}$ and the other $\boldsymbol{\lambda}_{\mathrm{s}}$ different. Measuring $\mathcal{D}_{1}=\left\{p_{4}\right\}$ or $\mathcal{D}_{2}=\left\{p_{3}, p_{5}\right\}$ the system is observable. If $w\left(\mathcal{D}_{2}\right)<w\left(\mathcal{D}_{1}\right)$ then the optimal solution is $\mathcal{D}_{2}$, even if $\left|\mathcal{D}_{2}\right|>\left|\mathcal{D}_{1}\right|$.

Proposition 10. Optimal cost solution need not to be of minimal cardinality.

Definition 11. The subnet $\left\langle t_{a}, p_{a}, t_{b}, p_{b}, \ldots, t_{y}, p_{y}\right.$, $\left.t_{z}, p_{z}\right\rangle$ and the arcs connecting those places and transitions is an incoming branch of place $p$ if:

- $|\bullet p|>1$

- $t_{a}^{\bullet}=p, p_{a}^{\bullet}=t_{a}$

- ...

- $\bullet_{z}=\emptyset$ or $\left|\bullet p_{z}\right|>1$ or $\bullet^{\bullet}\left(p_{z}\right) \subseteq$ $\left\{p_{a}, p_{b}, \cdots, p_{y}\right\}$ 
Let $\mathcal{G}_{p_{i}}^{j}$ be the places belonging to the incoming branch number $j\left(j \leq\left.\right|^{\bullet} p_{i} \mid\right)$ of the attribution $p_{i}$. Evidently, only one place per $\mathcal{G}_{p_{i}}^{j}$ can belong to the optimal solution (if two places were observed, one could be deduced from the other so it would not be optimal).

In the same way we can define the final incoming branch of every place in $\mathcal{F}$ and $\mathcal{G}_{\mathcal{F}}$ all places belonging to these branches. Places $\mathcal{G}_{\mathcal{F}}$ are estimated using the final places, so can be removed from the sets $\mathcal{G}_{p_{i}}^{j}$.

Considering again the net in Fig.2(b), we have $\mathcal{G}_{p_{1}}^{1}=\left\{p_{3}, p_{1}\right\}, \mathcal{G}_{p_{1}}^{2}=\left\{p_{2}\right\}, \mathcal{G}_{p_{2}}^{1}=\left\{p_{5}, p_{1}\right\}, \mathcal{G}_{p_{2}}^{2}=$ $\left\{p_{4}, p_{6}, p_{1}\right\}$ and $\mathcal{F}=\emptyset$. Assume $\boldsymbol{\lambda}_{5}=\boldsymbol{\lambda}_{6}$ and the other $\boldsymbol{\lambda}$ s different. Measuring $p_{3} \in \mathcal{G}_{p_{3}}^{1}$ the system is not observable $\left(p_{1}\right.$ and $p_{2}$ can be evaluated, but the attribution in $p_{2}$ is not observable because $\left.\lambda_{5}=\lambda_{6}\right)$. So, considering $p_{1} \in \mathcal{G}_{p_{1}}^{1}$ is a waste of time because the system cannot be observable. For $\mathcal{G}_{p_{1}}^{2}$ the system is again not observable $\left(p_{2}\right.$ is an attribution and $\left.\boldsymbol{\lambda}_{5}=\boldsymbol{\lambda}_{6}\right)$. Measuring $p_{5} \in \mathcal{G}_{p_{2}}^{1}$, the system is observable and also, for $p_{4}$ and $p_{6}$ from $\mathcal{G}_{p_{2}}^{2}$.

Three places which make the net system observable were found: $p_{4}, p_{5}$ and $p_{6}$. If the minimum measuring cost of these places is $w\left(p_{6}\right)$ the solution can be this one. So, it is obvious that it is essential to check the observability for all places in a $\mathcal{G}_{p_{i}}^{j}\left(p_{6}\right.$ is not the first place of $\left.\mathcal{G}_{p_{2}}^{2}\right)$, but an important observation appears:

Proposition 12. If the net system is not observable measuring a place from a $\mathcal{G}_{p_{i}}^{j}$ then it is not observable for all places situated upstairs in $\mathcal{G}_{p_{i}}^{j}$.

The maximum number of places that can be in the optimal solution is $|\mathcal{F}|$ plus the number of incoming branches. The optimal observability becomes a SCP; taking one or zero places belonging to a $\mathcal{G}_{p_{i}}^{j}$, all sets should be evaluated. Two methods can solve the problem: starting with one place, then two and so on, or starting with the maximum number of places that can belong to a solution and going backwards until one place is taken.

Definition 13. A set of $k$ places $\mathcal{C}_{k}$ is called a set of admissible places if $\forall p_{1}, p_{2} \in C_{k}$ and $\forall \mathcal{G}_{p_{i}}^{j}$ we have $\left|\left\{p_{1}, p_{2}\right\} \cap \mathcal{G}_{p_{i}}^{j}\right| \leq 1$.

Proposition 14. Let $\mathcal{C}_{k}$ and $\mathcal{C}_{l}(k \geq l)$ be two sets of admissible places such that any place from $\mathcal{C}_{l}$ either is in $\mathcal{C}_{k}$ or there exists a path from it to a place in $\mathcal{C}_{k}$. If the net system is not observable measuring $\mathcal{C}_{k}$ then is not observable measuring $\mathcal{C}_{l}$.

Proof: Measuring $\mathcal{C}_{k}$ all places $\mathcal{C}_{l}$ can be evaluated and obviously, if the net system is not observable measuring $\mathcal{C}_{k}$ it is not observable measuring $\mathcal{C}_{l}$.

\subsection{Computation Algorithm}

The results presented in this section allows to develop an algorithm for the optimal observability.

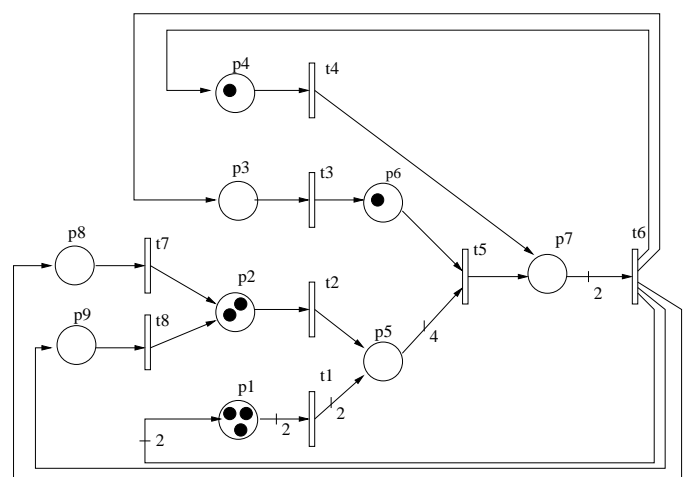

Fig. 3. Petri net modelling a table factory.

Proposition 14 suggests that the best way is to start with the maximum number of places that can belong to the solution in order to limit the number of searches. For every set of admissible places $\mathcal{C}_{k}$, the observability is checked, if the net system is observable then $\mathcal{C}_{k}$ is introduced into a set $\mathcal{S}$, if not, the set is introduced into $\mathcal{I}$ such that any other set $\mathcal{C}_{l}$ defined as in Prop. 14 will not be taken in the future.

$\operatorname{Input}\left\langle\mathcal{N}, \boldsymbol{\lambda}, \mathbf{m}_{0}\right\rangle$

Output Optimal solution of the observability

1. Transform a general net $\mathcal{N}$ into a JC-F by removing all synchronizations and merging all transitions in conflict.

2. Compute the set $\mathcal{F}$

if $\nexists$ attributions (Th. 9 applies) then

if $\mathcal{F}=\emptyset$ (it is a cycle) then

return one place with minimum cost.

else return $\mathcal{F}$ and $w(\mathcal{F})$.

end if

else

if $\mathcal{O}_{\mathcal{F}}$ (obs. matrix measuring $\mathcal{F}$ ) has full rank then return $\mathcal{F}$ and $w(\mathcal{F})$

else

Compute the sets $\mathcal{G}_{p_{i}}^{j}-\mathcal{G}_{\mathcal{F}}($ called $\mathcal{G})$

$\mathcal{S}:=\emptyset$

$\mathcal{I}:=\emptyset$

for all $i=|\mathcal{G}|:-1: 1$ do

for all $\mathcal{C}_{i}$ such that $\nexists \mathcal{C}_{j} \in \mathcal{I}$ verifying Prop. 14 do $r:=\operatorname{rank}\left(\vartheta_{\mathcal{C}_{i} \cup \mathcal{F}}\right)$

if $r=n$ then Insert $\mathcal{C}_{i}$ into $\mathcal{S}$ $\mathcal{S}=\mathcal{S}-\left\{\mathcal{C}_{j} \mid \mathcal{C}_{i} \subset \mathcal{C}_{j}\right\}$ end if else Insert $\mathcal{C}_{i}$ into $\mathcal{I}$

end for

end for

Get the set from $\mathcal{S}$ with minimum cost. end if end if

Therefore, all combinations of places that make the system observable are computed and the set with minimum cost is taken. The complexity of the algorithm remains exponential but in practice the number of covers has been considerably reduced. The places from one incoming branch are taken in the order that were defined $\left(p_{a}, p_{b}, \ldots\right.$, $p_{z}$ ) such that, if the system is not observable, we stop advancing in this incoming branch. 


\subsection{Case studies}

The small FMS presented in Fig. 3 was selected as an illustrative example and consists of three different machines to make table-legs, one $\left(t_{1}\right)$ which produces two legs at a time, and two $\left(t_{7}\right.$ and $\left.t_{8}\right)$ which make legs one by one; A machine $\left(t_{3}\right)$ to produce the table-boards; A machine $\left(t_{5}\right)$ to assemble four legs and a board; And a big painting line $\left(t_{6}\right)$ which paints two tables at once. The painting line has more capacity than the other machines, so more unpainted tables are brought $\left(t_{4}\right)$ from a different factory. The different products are stored in buffers: Table-legs are stored in $p_{5}$, the ones produced by the slow machines are first stored in $p_{2}$ and have to be taken to $p_{5}$ (operation $t_{2}$ ), boards are stored in $p_{6}$, and $p_{7}$ is devoted to the storage of unpainted tables. The rest of places contain work orders: Whenever the painting line finishes a couple of tables, it delivers work orders to the leg-makers, the board-maker, and the other factory. Moreover, $50 \%$ of the tables are assembled, and $50 \%$ are brought from the other factory, while $50 \%$ of the legs are produced by the fast leg-maker, and $50 \%$ by the slow ones, half and half.

Assume that we want to apply a desired control of the system and for this control the system observability is required. The number of clients in each buffer can be measured with a sensor, the cost of the sensor depends on the buffer. We want to know what is the minimum amount of money that we should invest to make the system observable.

Let us apply the algorithm presented in Subsection 5.4 assuming $\boldsymbol{\lambda}=\mathbf{1}$. After removing the synchronization, $\mathcal{F}=\left\{p_{5}, p_{6}\right\}$ and $\mathcal{G}_{\mathcal{F}}=$ $\left\{p_{5}, p_{6}, p_{3}, p_{7}, p_{4}\right\}$. Measuring $p_{5}$ and $p_{6}$ the system is not observable then we need compute the input branches that are: $p_{1} \rightarrow p_{4}, p_{2}, p_{8} \rightarrow p_{4}$ and $p_{9} \rightarrow p_{4}$ with $\mathcal{G}_{p_{5}}^{1}-\mathcal{G}_{\mathcal{F}}=\left\{p_{1}\right\}, \mathcal{G}_{p_{5}}^{2}-\mathcal{G}_{\mathcal{F}}=\left\{p_{2}\right\}$, $\mathcal{G}_{p_{2}}^{1}-\mathcal{G}_{\mathcal{F}}=\left\{p_{8}\right\}$ and $\mathcal{G}_{p_{2}}^{2}-\mathcal{G}_{\mathcal{F}}=\left\{p_{9}\right\} . \mathcal{C}_{4}^{1}=$ $\left\{p_{1}, p_{2}, p_{8}, p_{9}\right\}$ ensures the observability (together with $\mathcal{F})$ so $\mathcal{S}=\left\{\mathcal{C}_{4}^{1}\right\}$. In the second step, $\mathcal{C}_{3}^{1}=$ $\left\{p_{1}, p_{2}, p_{8}\right\}, \mathcal{C}_{3}^{2}=\left\{p_{1}, p_{2}, p_{9}\right\}, \mathcal{C}_{3}^{3}=\left\{p_{1}, p_{8}, p_{9}\right\}$ and $\mathcal{C}_{3}^{4}=\left\{p_{2}, p_{8}, p_{9}\right\}$ are introduced into $\mathcal{S}$ and $\mathcal{C}_{4}^{1}$ is removed. For $i=2$, the sets of places that ensure the observability are: $\mathcal{C}_{2}^{1}=\left\{p_{1}, p_{8}\right\}$, $\mathcal{C}_{2}^{2}=\left\{p_{1}, p_{9}\right\}, \mathcal{C}_{2}^{3}=\left\{p_{2}, p_{8}\right\}$ and $\mathcal{C}_{2}^{4}=\left\{p_{2}, p_{9}\right\}$. After this step, $\mathcal{S}=\left\{\mathcal{C}_{2}^{1}, \mathcal{C}_{2}^{2}, \mathcal{C}_{2}^{3}, \mathcal{C}_{2}^{4}\right\}$ and $\mathcal{I}=$ $\left\{\mathcal{C}_{2}^{5}, \mathcal{C}_{2}^{6}\right\}$, where $\mathcal{C}_{2}^{5}=\left\{p_{1}, p_{2}\right\}$ and $\mathcal{C}_{2}^{6}=\left\{p_{8}, p_{9}\right\}$. For $i=1$ the system is not observable for any set of admissible places so the optimal solution is $w\left(p_{5}\right)+w\left(p_{6}\right)+\min \left(w\left(p_{1}\right)+w\left(p_{8}\right), w\left(p_{1}\right)+\right.$ $\left.w\left(p_{9}\right), w\left(p_{2}\right)+w\left(p_{8}\right), w\left(p_{2}\right)+w\left(p_{9}\right)\right)$. The number of covers for the SCP in Section 4 is restricted to the number of different places combinations. To solve the problem is necessary to explore $2^{m}-$ $1=2^{9}-1=511$ combinations, while with this algorithm observability was checked only 16 times.

\section{CONCLUSIONS}

Optimal observability of continuous $P N s$ has been considered in this paper. Given a measuring cost per place, the problem is to find a cheaper set of places that makes the system observable. It can be viewed as a set covering problem, thus it is NP-hard; the purpose of this paper was to develop a covering algorithm capable to deal in practice with the problem. To do this, optimal observability was studied for different classes of $P N s$. In the case of JCA-F $P N s$ the problem can be solved in polynomial time. In a general case, which can be reduced to the JC-F PNs, the problem is exponential but the considerations presented in this paper reduce drastically the practical size.

In this paper we have focused on infinite servers semantics. It seems difficult to extend these results to finite servers, because this semantics provides much less information since the flow of a transition does not depend on the marking of its input places, as long as it is greater than zero or receives an input flow. However, more in depth study is needed. More work is also needed to understand how these results are related to observability of the underlying discrete systems.

\section{REFERENCES}

Ajmone Marsan, M., G. Balbo, G. Conte, S. Donatelli and G. Franceschinis (1995). Modelling with Generalized Stochastic Petri Nets. Wiley.

Garey, M.R. and D.S. Johnson (1979). Computers and Interactability: A Guide to the Theory of NP-Completeness. W. H. Freeman and Company.

Júlvez, J., E. Jiménez, L. Recalde and M. Silva (2004). On observability in timed continuous Petri net systems. In: Proc. of the 1st Int. Conf. on Quantitative Evaluation of Systems (QEST) 2004. Twente, The Netherlands. pp. 60-69.

Luenberger, D.G. (1971). An introduction to observers. IEEE Transactions on Automatic Control 16(6), 596-602.

Murata, T. (1989). Petri nets: Properties, analysis and applications. Proceedings of the IEEE $\mathbf{7 7}(4), 541-580$.

Ogata, K. (1995). Discrete-Time Control Systems, 2nd. ed.. Prentice Hall.

Recalde, L. and M. Silva (2001). Petri Nets fluidification revisited: Semantics and steady state. APII-JESA 35(4), 435-449.

Silva, M. and L. Recalde (2002). Petri nets and integrality relaxations: A view of continuous Petri nets. IEEE Trans. on Systems, Man, and Cybernetics 32(4), 314-327.

Silva, M. and L. Recalde (2004). On fluidification of Petri net models: from discrete to hybrid and continuous models. Annual Reviews in Control 28(2), 253-266.

Sinha, P. K. (1984). Multivariable Control: An Introduction. Marcel Dekker.

Teruel, E., J. M. Colom and M. Silva (1997). Choice-free Petri nets: A model for deterministic concurrent systems with bulk services and arrivals. IEEE Trans. on Systems, Man, and Cybernetics 27(1), 73-83. 